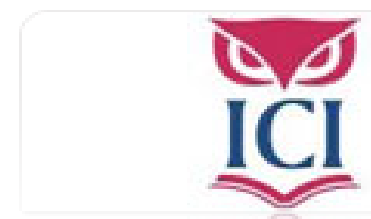

IUS. Revista del Instituto de Ciencias Jurídicas de Puebla A.C.

ISSN: $1870-2147$

revista.ius@hotmail.com

Instituto de Ciencias Jurídicas de Puebla A. C.

México

Guzmán Ávalos, Aníbal

La subrogación de la maternidad

IUS. Revista del Instituto de Ciencias Jurídicas de Puebla A.C., núm. 20, 2007, pp. 114-125

Instituto de Ciencias Jurídicas de Puebla A. C.

Puebla, México

Disponible en: http://www.redalyc.org/articulo.oa?id=293222932007

- Cómo citar el artículo

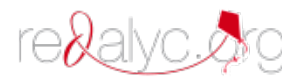

- Número completo

- Más información del artículo

Página de la revista en redalyc.org

Sistema de Información Científica

Red de Revistas Científicas de América Latina, el Caribe, España y Portugal

Proyecto académico sin fines de lucro, desarrollado bajo la iniciativa de acceso abierto 


\title{
LA SUBROGACIÓN DE LA MATERNIDAD
}

\author{
Anibal Guzmán Ávalos*
}

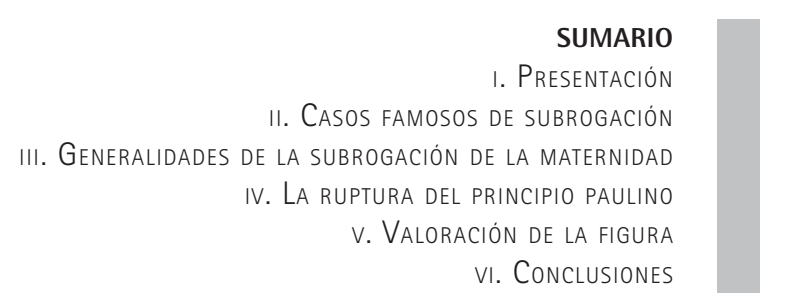

\section{RESUMEN}

El presente artículo sostiene que el desarrollo científico-técnico ha posibilitado desde los años setenta la procreación asistida, con lo cual se abrió un nuevo capitulo en la reproducción humana. Esto ha dado lugar a lo que se conoce como maternidad subrogada, cuestión en la que la relación maternidad-paternidad-filiación puede adoptar diferentes formas. De esta manera, en un contrato de este tipo pueden estar presentes los padres genéticos (proveedores del óvulo y esperma), la madre portadora o alquilada (que se limita a llevar el embarazo) y hasta los padres legales o educadores (se responsabilizan del cuidado del niño después de nacer). Así se puede hablar de madre genética, madre gestante y madre responsable. La fractura

\section{ABSTRACT}

The development scientific-technician has it facilitated from the years 70 the assisted procreation, with that which a new chapter opened up in the human reproduction. This has given place to what is known as surrogate maternity, question in which the relationship maternity-paternity-filiation can adopt different forms. Of this way, in a contract of this type can be present the genetic parents (suppliers of the ovum and sperm), the rented mother (that is limited to develop the pregnancy) and until the legal parents or educators (responsible for the boy's care). One can speak this way of genetic mother, pregnant mother and legal mother. The fracture in the conception-gestation brings I get diverse

* Doctor en derecho y profesor del área de derecho civil en la Universidad Veracruzana, México. 
en la concepción-gestación trae consigo diversas problemáticas de trascendencia jurídica, entre ellas las de filiación, las que han tenido soluciones distintas en los ordenamientos jurídicos que se han decidido a pronunciarse sobre el particular. El artículo aborda casos referentes en Estados Unidos, Francia e Inglaterra y la solución que se ha dado en el estado de Tabasco, en México. problems of artificial transcendency, among them those of filiation, those that have had different solutions in the juridical classifications that have decided have to be pronounced on the matter. The article approaches relating cases in United States, France and England and the solution that it has been given in the state of Tabasco in Mexico.

\section{i. Presentación}

En los últimos años ha sido muy notable el avance que se presenta en la ciencia médica y especialmente dentro del campo de la biogenética, existiendo así un gran auge en el panorama de la investigación y la experimentación, dándose con esto una gama de oportunidades en este campo al ser humano.

Con la procreación asistida se inaugura un nuevo capítulo en los avances técnicos médicos sobre el organismo humano, con lo que se quiere poner remedio a la incapacidad de engendrar en forma natural un hijo, mediante la terapia de favorecer la reproducción sin el encuentro físico entre el hombre y la mujer. Es cierto que dichos avances crean problemas de dramatismo o de asombro jurídico que hacen plantear interrogantes a los conflictos de pretensiones o derechos de filiación que se crean ante el concurso, en algunas de las técnicas, de varios sujetos en el proceso de fecundación, cuya participación en principio, es con distintos intereses, pero que al final puede revestir uno solo: la filiación del hijo.

\section{Casos famosos de subrogación}

En 1975 se publicó en los Estados Unidos un anuncio de un periódico, a petición de una pareja estéril, donde solicitaban una mujer para que fuera inseminada artificialmente ofreciendo retribución por ese servicio. De esa fecha en adelante concretamente en el año de 1982 en el estado de California aparecen anuncios de madres portadoras con nombre de treinta de éstas, como también de sesenta y cuatro personas ansiosas de tener un hijo por dicho método. 
Sin embargo, el caso más famoso es el concertado entre Mary Beth Whitehead, una mujer casada de unos treinta años, madre de dos hijos y el matrimonio Stern (formado por William Stern, con una edad sobre los cuarenta años, hijo de unos sobrevivientes del Holocausto y destinado a ser el último descendiente, ya que su mujer sufría una enfermedad que con el embarazo se le hubiera agudizado y no estaba dispuesta a aceptar los riesgos de la gestación y el parto). El contrato comprendía el pago de 10,000 dólares y todos los gastos médicos. Dicho convenio lo firmó la señora Whitehead con el fin de "dar el más cariñoso don de felicidad a una pareja desafortunada" y de aprovechar la suma de dinero para poder proveer en un futuro los estudios de sus dos hijos. ${ }^{1}$

Los términos del contrato, como se reproducen en los hechos de la sentencia, fueron que la señora Whitehead se obligó a intentar la concepción a través de la inseminación artificial, de llevar a cabo el embarazo, parir y entregar el niño o niña al señor Stern, renunciando a sus derechos de potestad y dando por hecho que este acto era en interés del menor. Por otro lado, se convino que el nombre de los Stern aparecería en el certificado de nacimiento; que la señora Whitehead asumiría el riesgo del embarazo y del parto y se sometería a un examen psiquiátrico, a costa del señor Stern. El derecho de dar el nombre al niño(a) le correspondía a Stern. Si eventualmente muriera el señor Stern el niño(a) se le confiaría a su esposa. La señora Whitehead se obligó a no abortar y someterse a examen médico y si el feto resultaba con anormalidad genética o congénita la señora Whitehead se obligaba a abortar a solicitud del señor Stern. ${ }^{2}$

El 27 de marzo de 1986 en el Monmouth Medical Center de Long Branch, New Jersey, la niña nace. Sería mundialmente famosa como "Baby M". Ese mismo día el padre biológico y contractual se trasladó al hospital para ver a la niña y se percata que contrariamente a los acuerdos tomados, ella tiene el nombre de la familia de la madre natural: Sara Elizabeth Whitehead. Primer incumplimiento, porque lo convenido era que la niña habría debido ser llamada como hija de William Stern. De todos modos solicitó que, según los acuerdos, la niña le fuera entregada. Mary Beth Whitehead, arrepentida de la obligación asumida, lo rechazó y se da a la fuga por todo el país. ${ }^{3}$

' Carmel Shalev, Nascere per contratto, Giuffrè Editore, Milano, 1992. p. 5.

${ }^{2}$ Vincenzo Franceschelli, "Verso una paternità contrattuale: famiglia artificiale e diritto privato", Il diritto della famiglia e delle persone, Anno xv, Giuffrè Editore, Milano, pp. 1160-1161.

${ }^{3}$ Ibid., p. 1161. 
La verdad es que estos acontecimientos encierran una triste historia de relaciones humanas, antes que un caso jurídico, pero son todavía más tristes, quizá, los acontecimientos judiciales.

Los Stern resolvieron acudir a la justicia para hacer valer el acuerdo de subrogación, conociendo en primera instancia el juez Harvey Sorkow, para Hackensack (New Jersey), condado de Bergen, que entregó la custodia de la criatura a los Stern y que en su sentencia determinó que el contrato era válido y legal, terminando con todos los derechos de la portadora. Se revela constante la preocupación por el interés de la menor, que se encuentra en el centro del caso judicial, pues la sentencia reza que cuando la corte de justicia constreñida a elegir entre los derechos de los progenitores y el bien de la niña, la elección es y debe ser el bien de la niña y su prevaleciente interés. Dicha sentencia fue recurrida por la señora Whitehead, declarando el Tribunal Supremo del Estado la nulidad del contrato por ir en contra de la ley y el orden público; reintegra sus derechos a la madre pero confirma la decisión de la corte inferior, acerca de otorgar la custodia al padre (Stern), sobre la base del interés de la menor. ${ }^{4}$

Si se evalúa la sentencia desde los parámetros medios de la sociedad norteamericana, es claro que la balanza de la justicia debía inclinarse a favor de la pareja Stern. Por un lado, el matrimonio Whitehead carecía de una formación intelectual, su nivel económico era inferior al de los Stern, se habían mudado de casa por lo menos doce veces en los últimos años, aparentemente la mujer tiene un papel dominante en la familia porque el hombre es alcohólico. Por su parte, el matrimonio Stern estaba constituido por dos personas con doctorados, económicamente más favorecidos y, en apariencia, con mayor estabilidad.

A fines de 1986 existían ya más de cien casos conocidos de madres portadoras en los Estados Unidos. Entre ellos está el caso de Anna Johnson, madre portadora que después del alumbramiento del hijo reclama compartir la custodia, extremo a que se opusieron los padres genéticos, matrimonio formado por Mark y Crispina Colvert. Esta última, ante la imposibilidad de tener descendencia al haber sufrido una intervención que le impedía conservar el feto en su seno, optó por utilizar los servicios de una madre subrogada, Anna Johnson. Después de la fecundación in vitro del óvulo de Crispina con el semen de su esposo, el embrión resultante

\footnotetext{
${ }^{4}$ Véase Carmel Shalev, Op. cit. p. 7-11; J. M. Martínez Pereda Rodríguez y J. Massigoge, La maternidad portadora, subrogada o de encargo en el derecho español, pp. 28-31; Vincenso Franceschelli, Op. cit. p. 1161.
} 
fue implantado en el útero de Anna, quien se negó a entregar al niño, alegando que durante el embarazo se había establecido un lazo especial entre ambos, por lo que decidió reclamar la custodia compartida, pero los Colvert no estaban dispuestos en modo alguno a hacerlo.

El juez de la Corte Superior del Condado de Orange resolvió originalmente la controversia permitiendo a Anna Johnson visitar tres horas diarias al recién nacido, y dando la custodia legal a los padres genéticos. Tras las declaraciones de la portadora a la revista Los Angeles Times, en el sentido de que genéticamente nada tenía que ver con el feto, que si por el contrario hubiera sido su óvulo, habría sido diferente, y por lo que hace a la cuestión emocional, después introducida, resultaba un "cuento", por lo que en la revisión del caso el juez de Santa Anna (California) otorgó la custodia del pequeño Chistopher, de dos meses de edad a sus padres biológicos, Mark y Crispina Colvert, negando además cualquier derecho de visita a la madre de alquiler, argumentando que esto tenía como objetivo evitar confusión afectiva y emocional al pequeño. Una situación de dos madres sería muy confusa para la criatura y los derechos de visita y custodia parcial "facilitarían la extorsión emotiva y financiera", según indicó en la sentencia. ${ }^{5}$

La maternidad subrogada se ha establecido en otros países, como en Gran Bretaña, en donde el primer caso fue el de Mary Stewar, que dio a luz y entregó después la criatura al padre biológico, según se había acordado. Mary Stewar atendió a un anuncio publicado en un diario por una pareja estéril.

En Francia el fenómeno de las madres sustitutas comenzó su desarrollo hacia 1982, con la fundación del doctor Sacha Geller, director del Centro de Investigación de Técnicas de Reproducción, primera asociación destinada a poner en contacto matrimonios y parejas estériles con futuras madres portadoras. La asociación determinaría la creación de otras dos: Sainte Ara y Alma Mater, además de Les Cigognes, la cual desaparecería pronto. Como consecuencia de tal actividad, en 1987 se dieron más de cien nacimientos mediante tales técnicas, habiendo gestionado Alma Mater sesenta y seis de ellos.

El caso de las gemelas Cristine y Magali, de La Grande Motte, localidad de Hevearlt, se hizo igualmente patente en todo el mundo. Cristine fue inseminada artificialmente con esperma de su cuñado, Denis Sevault,

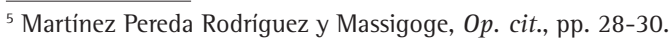


marido de su hermana gemela. La portadora dio a luz un niño en 1983 y manifestó haber aceptado el embarazo y parto por el profundo amor que sentía por su hermana.

En 1985 en una clínica de Montpellier, Francia, Patricia Lavisse, de 22 años, inseminada artificialmente con semen del marido de la pareja que la contrató, dio a luz una niña. El caso revistió trascendencia por tratarse del primer supuesto divulgado de operación cremástica en Francia. La señora Lavisse recibió 50,000 francos por el servicio, además de otros valiosos regalos, siendo entregada la niña al matrimonio contratante sin resistencia de la portadora. ${ }^{6}$

\section{Generalidades de la subrogación de la maternidad}

Se utiliza en los casos de falta o malformación uterina. Se realiza a través de la fecundación in vitro y transferencia del embrión o inseminación artificial y consiste en contar con los servicios de una mujer para que lleve el embarazo con la intención de entregar el niño o la niña al nacer a las personas que los han encargado. Puede revestir formas diversas de la maternidad-paternidad-filiación y pueden intervenir hasta tres madres y dos padres: por un lado los padres legales o educadores, no genéticos, que se responsabilizan del cuidado del niño después de nacer; por otro, los padres genéticos: los proveedores del óvulo y esperma; finalmente la madre portadora o alquilada, que se limita a llevar el embarazo. La mujer inseminada es una madre sustituta que ha prestado su útero para que pueda lograrse en él lo que no podría realizar una mujer estéril.

Se ha definido como: "el contrato mediante el cual una mujer se obliga, frente a una pareja estéril, mediante una compensación, a llevar a término un embarazo, haciéndose fecundar artificialmente con el semen del marido de la pareja o su embrión y entregar al recién nacido inmediatamente después del parto a los cónyuges, los que asumirán cualquier derecho y deber frente al niño".

Este contrato puede encuadrarse dentro de los atípicos, siempre en los límites de la autonomía de la voluntad, a título oneroso, aunque no se excluye que sea a título gratuito.

Quienes recurren a esta práctica son mujeres que cuentan con gónadas

\footnotetext{
${ }^{6}$ Ibid., pp. 35-36.

${ }^{7}$ Donata Clerici, "Procreazione artificiale, pratica della surroga e contratto de maternità: problemi giuridici”, Il diritto di famiglia e delle persone, p. 1015.
} 
normales capaces de ovular pero que por alguna causa, no pueden lograr un embarazo o éste no puede llegar a término (matriz infantil, aborto recurrente, malformaciones uterinas). El primer nacimiento concebido por FIV-TE, ocurrió el 13 de abril de $1986 .{ }^{8}$

\section{LA RUPTURA DEL PRINCIPIO PAULINO}

No hay duda de reconocer a la madre que ha parido, pero ahora que hay fractura entre concepción-gestación, esa certeza que era el presupuesto de la norma jurídica ya no parece ser tan cierta; por el contrario, cae por tierra aquel viejo principio paulino.

Hay que analizar detenidamente este supuesto, ya que puede verse la participación de hasta tres mujeres: la primera, que produce y dona el óvulo, sin cuya participación no se habría dado inicio al proceso generativo (a ella se le dado el nombre de madre genética); la segunda, que hace que el germen de vida contenido en el gameto se transforme en un ser humano, alimentando y protegiendo dentro de su cuerpo al embrión (biológico gestacional); la tercera, que no participa en ese proceso de procreación, pero es la madre educacional y de deseo (afectiva o social). La primera transmite a través del embrión los propios caracteres al nasciturus, así como los del padre y es también parte formativa de la descendencia, se dice que es la verdadera madre; ${ }^{9}$ la segunda, que aporta un componente psicológico y fisiológico que supone la relación constructiva e intensiva del periodo de gestación que contribuye, decisivamente, a que el proceso de formación y

\footnotetext{
${ }^{8}$ José Gorozpe y Ernesto Gómez, "Reproducción asistida. Alternativas", Ginecología y obstetricia de México, Vol. 61, Federación Mexicana de Asociaciones de Ginecología y Obstetricia, México, agosto de 1993, p. 217.

${ }^{9}$ Ésta es la posición de Valente Simi, "Manipulazioni genetiche e diritto", en Quaderni di iustitia, 34, Unione Giuristi Cattolici italiani, Giuffrè Editore, Roma, 1986, pp. 212-213, quien sostiene que "cuando resulta que el embrión formado por una pareja, distinta de la mujer en que se desarrolla la gestación, a través de un contrato, como se puede negar la justa veneración y prevalencia del embrión, así felizmente ilustrada en la relación Lombardi, ¿cómo se puede pretender negar en realidad la verdadera descendencia hereditaria? Los cambios científicos crean una verdadera figura nueva, no considerada por el legislador, la solución de la cual el intérprete podrá recabar de los elementos probados, dados, recabados y de los principios generales del derecho, que tienden a reconocer la prevalencia de la descendencia real, derivada del embrión generatore, esto es la realidad y no la simple apariencia de la relación filial, cuando resultado de los hechos aparece la verdad de la fecundación-gestación desligada..." Maria Costanza, en "Legislazione e fecundazione artificiale", Il diritto de famiglia e delle persone, Anno xvl, Giuffrè, Milano, 1987, pp. 1021-1026, dice: "la maternidad puede ser jurídicamente atribuida a aquella que ha determinado el patrimonio genético del nasciturus, más que aquella que ha contribuido a su desarrollo en la etapa fetal. Si bien la gestación no es un momento indiferente en la vida del individuo y en su personalidad, es menos incisiva que el momento procreativo donde se imprimen los caracteres fundamentales de la persona".
} 
desarrollo de la vida se consolide en el nacimiento del ser humano $;^{10}$ y la tercera, que asume la maternidad con voluntad y autorresponsabilidad e influye decisivamente en los hijos, en el desarrollo de su personalidad, de su inteligencia y de su sensibilidad social. ${ }^{11}$

A veces esas tres funciones recaen en dos mujeres, en combinaciones diferentes: a) la comitente aporta el gameto, además la decisión de que nazca ese hijo concreto "suyo", y la otra es la madre gestante; b) junto a la comitente colabora la gestante, que pone también gameto propio. Si una de aquellas tres, o de estas dos, ha de ser madre, lo único claro es que ninguna de ellas es la madre que Paulo daba siempre como cierta, ni la suya es la maternidad histórica, clásica, esa institución jurídica y social perfectamente acuñada y conocida.

El problema puede existir cuando se rompe el nexo entre gravidez y fecundación; es decir, cuando una de la mujeres participantes en el proceso reproductivo haya aportado el óvulo ya fecundado con el espermatozoide de su marido o compañero y hay transferencia del embrión con dicho material genético o con ovocitos de otra mujer o únicamente con el gameto del marido de la pareja comitente a otra distinta, "la portadora".

El problema es discutible a fin de determinar cómo se establecería el lazo filial, por lo que debe de realizarse una valuación comparativa de los distintos comportamientos y contribuciones de dichas mujeres, valorando los elementos en juego: el biológico, el voluntario y el socio-afectivo constituido por la posesión de estado, que constituirían los elementos fundamentales de la filiación. ${ }^{12}$

De lege data siempre será madre la que ha gestado, con independencia de quien donó el material genético, ya que no es fácil la atribución de la maternidad a quien no ha parido. ${ }^{13}$ Amén que la paternidad y la maternidad en México no son renunciables, ni pueden ser objeto de transacción. Por otra parte en las leyes punitivas del país se tipifica que incurre en delito contra el estado civil de las personas, quien atribuya a un niño recién nacido a mujer que no es su madre o usurpe el estado civil de otro, con el

\footnotetext{
${ }^{10}$ Ésta es la posición mayoritaria tanto por los ordenamientos jurídicos, como por las comisiones, recomendaciones, doctrina y proyectos de leyes relacionados con la materia.

${ }^{11}$ María Dolores Pelayo Duque, Comisión especial de estudio de la fecundación in vitro y de la inseminación artificial humanas, Sesión 14 de noviembre de 1985, Diario de Sesiones del Congreso de Diputados, No. 367, Madrid, p. 11179.

${ }^{12}$ En este sentido Monique Bandrac, "Reflexions sur la maternité”, Dalloz-Sirey, Paris, 1985, p. 29.

${ }^{13} \mathrm{Y}$ es aquí donde debe de intervenir el legislador para modificar la identificación de la maternidad porque ello no crea fracturas con el ordenamiento ni con los principios constitucionales, según señala Maria Costanza, Op. cit., pp. 1021-1026.
} 
fin de adquirir derechos de familia que no le corresponden. No hay vuelta de hoja: madre será la que ha parido.

De llevarse a cabo la práctica de este tipo de procreación, las partes que hayan celebrado algún acuerdo de sustitución de maternidad, para conseguir los objetivos trazados en el mismo y lograr la filiación del hijo, simplemente se constituyen en franco fraude a la legislación respectiva.

El sistema actual en caso de maternidad subrogada deja fuera de la relación filial a la "madre genética", a la que sólo aporta el óvulo para la fecundación, si no es la misma que la gestante o "madre biológica". ${ }^{14}$ Lo cierto es que, a excepción del Código Civil del Estado de Tabasco, en general en la legislación nacional la maternidad subrogada no tiene existencia jurídica y, por el contrario, puede considerarse, incluso, prohibida porque el objeto del acuerdo es el ser humano, mismo que se encuentra fuera del comercio. Quizá el remedio consistiría en instrumentar una legislación más flexible, que prevenga en su caso, la adopción por parte de la madre genética; de una forma simplificada y adecuada que permita la adopción inclusive del nasciturus ${ }^{15}$ o del embrión, como una especie de adopción prenatal, pues el hijo podría adoptarse desde su concepción. ${ }^{16}$ Como ahora lo regula la legislación tabasqueña. Sin embargo, el principio básico sigue vigente: el ser humano está fuera del comercio, por tanto, en el resto de la legislación nacional, la maternidad subrogada es ilícita.

La única manera de evitar los problemas derivados de la maternidad subrogada es aceptar y definir de manera expresa tal figura que no existe y no tiene cabida en el derecho mexicano, y por lo tanto se prohíba cualquier negocio jurídico realizado al respecto y se considere nulo de pleno derecho, resolviendo los problemas de filiación en atención a los principios y supuestos que sostienen a la institución de la filiación sin dar solución al problema para los casos que aún con esa sanción se lleven a cabo.

\footnotetext{
${ }^{14}$ Donata Clerici, Op. cit., pp. 1018-1019.

${ }^{15}$ Michelle Gobert, "Audiencia pública sobre los problemas jurídicos y éticos de la genética humana, con especial referencia a los problemas relacionados con la ingeniería genética”, Parlamento Europeo, Problemas éticos y jurídicos de la manipulación genética y de la fecundación humana. Comisión de asuntos jurídicos y de derechos de los ciudadanos. Oficina de publicaciones oficiales de las comunidades europeas. Celebradas en Bruselas, del 19 a 21 de marzo de 1986. p. 135; Gutiérrez Merino, "Los consentimientos relevantes y las técnicas de reproducción asistida, 1 Congreso mundial vasco, La filiación a finales del siglo XX; Problemática planteada por los avances cientificos en materia de reproducción humana, Ed. Trivium, Madrid, 1988. p. 428.

${ }^{16}$ Rubio, Comisión especial de estudio de la fecundación in vitro y de la inseminación artificial humanas, Sesión 23 octubre de 1985, Diario de Sesiones del Congreso de Diputados, No. 346, Madrid, p. 10650.
} 


\section{VALORACIÓN DE LA FiguRA}

La posición de rechazo de la sustitución de la maternidad es mayoritaria, tanto legislativa como doctrinariamente. ${ }^{17}$ Sin embargo, como he manifestado en otros trabajos similares, entre los elementos fundamentales de la filiación se incluye el de la "voluntad" y si se sostiene, para el caso de determinar la paternidad, en los supuestos de donador de semen, la filiación con el varón que otorga su consentimiento. Entonces, ¿por qué se tendría que cambiar el criterio para determinar la maternidad, ya que la mujer comitente desea el hijo para ella y la portadora no pretende hacer valer el título de la atribución del Estado y no busca ni desea en principio hijo propio, y ni siquiera proporciona óvulo, ni hay acto sexual? Es decir: ¿aquí la voluntad no tendrá un significado relevante? Es cierto que no es lo mismo donar un gameto que portar un hijo; los supuestos son distintos, el vínculo que se crea entre el producto de la concepción y la madre es superior a una simple donación de gametos. Aquí se muestra ya en todo su esplendor el lazo maternal que une a la portadora con el hijo. Es por ello que ha habido incumplimientos de convenios que han traspasado las fronteras y han sido de dominio público.

Sin embargo, si se quiere sostener en este tipo de procreación que hay que hacer referencia al elemento de voluntad de querer asumir la maternidad, elemento necesario para atribuir la filiación, en congruencia con el derecho a procrear, como parte de los derechos fundamentales, no se puede negar que una mujer preste su útero a otra para llevar a cabo un embarazo. Las mujeres que intervienen son mayores de edad, con un discernimiento propio para decidir la maternidad y si se les impide, simplemente se les priva del derecho a la maternidad y a las sustitutas la acción humanitaria y solidaria con quienes no pueden tener hijos.

Bajo esta óptica, hay voces que pugnan por que la maternidad se atribuya a la mujer comitente, la que quiso ser madre legal y así lo pactó en el convenio, sin distinguir inclusive si aportó los gametos o no. Si bien es cierto que rompe con la máxima paulina, también es cierto que no hay impedimento para otorgar la maternidad a quien no dio a luz, pues se trata de una nueva situación que no tiene que solucionarse siguiendo los mismos criterios que se siguen con la maternidad natural; tomando en

${ }^{17}$ Véase Aníbal Guzmán Ávalos, Inseminación artificial y fecundación in vitro: Un nuevo modo de filiación, Universidad Veracruzana, Xalapa, 2000. 
consideración: a) la voluntad de querer el hijo; b) la voluntad de la madre sustituta de aceptar llevar el embarazo y la voluntad de renunciar a cualquier derecho sobre el niño; c) no cabe alegar que la gestante adquiere motivos sentimentales con el niño que ha llevado en su seno, pues si en realidad éstos existen, lo cierto es que esos detalles los ha conocido desde el momento de la celebración del contrato; d) la misión de la madre sustituta es dar a luz un hijo y posteriormente entregarlo a la madre comitente; e) en congruencia con la inseminación artificial heteróloga y fecundación in vitro, en donde no se otorga la paternidad-maternidad a los donadores de gametos, sino a las personas que han deseado y querido un hijo para ellos, también no se debe otorgar la filiación a la portadora, ya que como en aquellos casos, sólo interviene para hacer posible el nacimiento de un niño, aun cuando su actividad acá sea más prolongada; y f) porque se dan casos que la madre sustituta una vez que ha dado a luz al hijo, no desea ni quiere para ella ese niño que ha nacido y consecuentemente originaría que la ley le atribuya la maternidad de un hijo no deseado, que sólo lo gestó para otra. ${ }^{18}$

Hay quienes siguiendo este criterio, lo hacen más restringido, pues aceptan que la gestación sustituta con aportación de material genético de la pareja comitente debería autorizarse, siempre que se lleve a cabo sin intermediarios y en forma gratuita. ${ }^{19}$ Por lo que se debe buscar un mecanismo que asegure la maternidad genética; que en su caso, dé fuerza legal a los acuerdos que se estipulen entre las partes, ya que la identidad con el hijo se consigue con el contacto sano y afectivo con los padres, sean o no genéticos. ${ }^{20}$

Hay quien señala que la solución para determinar la maternidad es aquella por la cual el estatus debe ser reconocido a quien asume en sí más comportamientos a los tres posibles: "madre genética", "madre gestante", "madre responsable", o en su caso, se considera prevalece la madre gené-

${ }^{18}$ Véase Carmen Hernández lbañez, "La ley de 22 de noviembre de 1988 sobre técnicas de reproducción asistida: consideraciones en torno a la fecundación post mortem a la maternidad subrogada”, en Actualidad Civil, No. 48, semana 26 de diciembre 1988-1 de enero de 1989, pp. 3044-3045.

${ }^{19}$ Gutiérrez Merino, Op. cit., p. 428.

20 "Se tiene que asegurar un mecanismo por el que el contrato de subrogación sea un firme asegurador de la maternidad genética. ¿Cómo hacer para dar fuerza legal a ese contrato? No se puede esperar a una interpretación personal del juez que lleve el caso. La identidad se consigue con el contacto sano y afectivo con los padres (genéticos o no). Si el hijo conoce que ha nacido de inseminación artificial y su desarrollo psicológico ha sido bueno lo integrará como un acto de amor y de confianza hacía su persona de sus padres. Hay hijos de matrimonios que tienen conflictos de paternidad y buscan a su padre en una figura política o religiosa."Ver Pereda-Caballero, Op. cit., pp. 4-6. 
tica y a la madre de útero se le reconoce sólo un título preferencial para obtener en adopción el hijo procreado. ${ }^{21}$

No habría duda de atribuir la maternidad a la mujer que ha proporcionado el óvulo, ha gestado y finalmente ha parido. Asimismo, la mujer que tiene dos contribuciones tiene mayor derecho a atribuírsele la maternidad que la que sólo tiene una; esto es, que madre sería la que ha proporcionado el óvulo y desea el hijo (prevaleciendo sobre aquella que sólo ha gestado); o sería también la que ha gestado y desea el hijo (prevaleciendo sobre la mujer que sólo proporciona el óvulo); pero nunca la que sólo desea el hijo, sin ninguna otra contribución. ${ }^{22}$

Concluyendo: en congruencia con la igualdad del hombre y la mujer y el derecho de libertad de procreación, habrá que aceptar la atribución de la maternidad a la madre comitente, cuando así se haya expresado la voluntad en documento indubitable entre las participantes del acto procreativo, quizá manejando límites como que los gametos sean cuando menos de uno de los comitentes o por el mayor interés del hijo a nacer, como en el caso de las resoluciones de los casos famosos en esta materia.

Volver al Índice >>

\footnotetext{
${ }^{21}$ María Teresa Carbone, "Maternità, paternità e procreazione artificiale", Il diritto di famiglia e delle persone, Anno xxıl, Giuffrè, Milano, 1993, p. 881.

${ }^{22}$ En este sentido Fernando Santosuosso, Il diritto di famiglia e delle persone, Anno xvi, Giufre, Milano, 1987, pp. 55 y 126.
} 